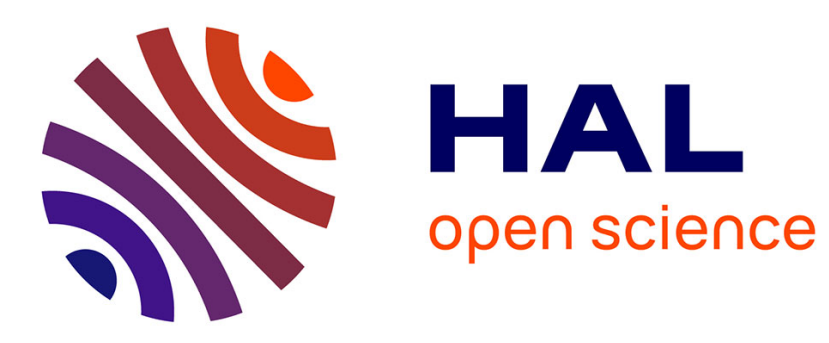

\title{
DC transformer for DC/DC connection in HVDC network
}

Jimenez Carrizosa, Abdelkrim Benchaib, Pedro Alou, Gilney Damm

\section{To cite this version:}

Jimenez Carrizosa, Abdelkrim Benchaib, Pedro Alou, Gilney Damm. DC transformer for DC/DC connection in HVDC network. 15th European Conference on Power Electronics and Applications (EPE 2013), Sep 2013, Lille, France. (elec. proc.), 10.1109/EPE.2013.6631774 . hal-00924024

\section{HAL Id: hal-00924024 \\ https://hal.science/hal-00924024}

Submitted on 18 Mar 2014

HAL is a multi-disciplinary open access archive for the deposit and dissemination of scientific research documents, whether they are published or not. The documents may come from teaching and research institutions in France or abroad, or from public or private research centers.
L'archive ouverte pluridisciplinaire HAL, est destinée au dépôt et à la diffusion de documents scientifiques de niveau recherche, publiés ou non, émanant des établissements d'enseignement et de recherche français ou étrangers, des laboratoires publics ou privés. 


\title{
DC transformer for DC/DC connection in HVDC network
}

\author{
M. Jiménez Carrizosa ${ }^{1}$, A. Benchaib ${ }^{2}$, P. Alou ${ }^{3}$, G. Damm ${ }^{4}$ \\ LSS/ SUPELEC ${ }^{1}$, ALSTOM GRID ${ }^{2}$, Centro de Electrónica Industrial/UPM ${ }^{3}$, Laboratoire IBISC ${ }^{4}$ \\ Miguel.Jimenez@1ss.supelec.fr, abdelkrim.benchaib@alstom.com, pedro.alou@upm.es, gilney.damm@ibisc.fr
}

\section{Acknowledgments}

This work is supported by WINPOWER project (ANR-10-SEGI-016).

\section{Keywords}

$<<$ DAB $>>,<<$ multilevel $>>,<<$ nonlinear control $>>,<<$ HVDC $>>,<<$ Plug \& Play $>>$.

\begin{abstract}
This paper presents the modeling and control of a multilevel DC/DC bidirectional converter suitable for medium voltage and power applications, with a special interest in wind-power applications. The proposed multilevel topology has a modular structure constituted by base DC/DC converter cells. The multilevel converter is consequently based on Dual Active Bridge (DAB). The overall control of the $\mathrm{DC} / \mathrm{DC}$ converter is achieved by using a nonlinear control based in Lyapunov theory.
\end{abstract}

\section{Introduction}

World energy demand is progressively growing while fossil fuels deplete. Moreover, renewable energy sources are often located far away from consumption centers (e.g. offshore wind farms). For this reason, power delivery through long distances is getting increasingly important. From the beginning of $20^{\text {th }}$ century and up to nowadays, electric power has been predominantly transmitted and distributed in AC due to the unavailability of well-suited DC/DC converters. However, the emergence of a wider range of power electronics devices is contributing to revitalize the research on DC transmission networks. DC grids present several advantages in comparison with AC grids: in DC networks there are not reactive power, so their transmission capacities are increased, they also produce fewer losses than AC grids, the weight and cost of components is lower (fewer cables), and the efficiency is higher [1, 2, 3, 4]. It is in this context where DC/DC converters play a decisive role. Since energy is generated at low voltages and highly efficient devices are needed to increase these voltages to higher values. Furthermore, these converters have to be bidirectional. This paper discusses the topology and the control of a DC/DC bidirectional converter valid for the integration of an offshore wind farm in a High Voltage Direct Current (HVDC). Until developed of VSC (voltage source converters), in HVDC systems were used the CSC (current source converters) to carry out the inversion and rectification. VSC converters use IGBTs and CSC use thyristors. VSC-HVDC systems present many advantages compared with CSC-HVDC systems. Some of them are: simultaneous control of active and reactive power, they create any phase angle or voltage magnitude (within certains limits), and they do not change voltage polarity when power direction is changed. Also CSC systems need communication between converters and in VSC systems it is not required [5]. We will suppose in this paper that we will employ VSC-systems, but the goal of this paper is to developed the DC/DC converter. The proposed converter would be located according to Figure 1 .

The paper is outlined as follows: Section I presents the introduction and the background information on the study. Section II presents an overview of existing high-power DC/DC converter topologies. Then 


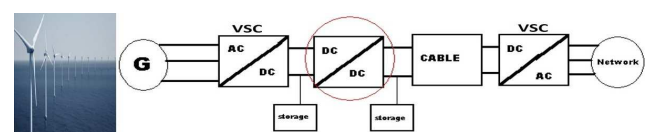

Figure 1: General scheme of installation.

the most suitable converter cell (DAB type) is chosen as a base-cell for the multilevel converter. Section III presents the modeling and control of a single DAB converter cell. The proposed control is a robust nonlinear control based on Lyapunov theory. Section IV presents the modeling and the control of the whole multilevel structure. In Section $\mathbf{V}$ the conclusions are explained.

\section{DC/DC Bi-Directional Converters. State of the Art}

As found in the literature, this report will show 3 main topologies which should be good candidates for our purpose. They are: DAB, SRC (Series Resonant Converter) and DHB (Dual Half Bridge). Such converter with its associated control algorithm will be considered as the basic block of a multilevel converter for HVDC applications. Our aim is to obtain a building block DC converter based on the so-called "Plug \& play" philosophy.

\subsection{Dual Active Bridge (DAB)}

The dual active bridge, $\mathrm{DAB}$, is comprised of two full bridge converters each connected to one side of the transformer, which will provide galvanic insulation (necessary to protect against unforeseen circumstances) [6]. DAB control will take place with rectangular modulation. This topology can enable a ZCS (zero current switching) in the high voltage side of the converter. An inductance L, which is used as a component of energy transfer, is placed in series with the transformer. The scheme of this structure is shown in Figure 2a. Medium frequency transformer is used [7,8] . It provides insulation and the step up/down of the different voltage levels. This transformer is based on a single magnetic core with windings in both legs of the core. Using this type of transformer the ratio weight/volume could be improved. It works at frequencies around 10/20 kHz [7]. For this type of converter SiC-JFETs can be used $[9,10,11,12]$, but the object of this paper is not study in depth the semiconductors and the internal transformers.

\subsection{Series Resonant Converter (SRC)}

This converter consists of two full bridges interfaced by a transformer with a series resonant tank composed of an inductor $\mathrm{L}$ and a capacitor $\mathrm{C}$. The SRC converter represents an attractive alternative for the HV-ZCS current modulation [6]. Piecewise sinusoidal current waveforms are obtained through the transformer with this resonant tank. The scheme structure is shown in Figure 2b:

\subsection{Dual Half Bridge (DHB)}

Dual half bridge (DHB) is a popular topology among phase-shift ZVS bidirectional DC-DC converters [13]. To achieve ZVS a is adopted for all switching devices without auxiliary switch devices in either direction of power flow, and therefore it enables the high switching frequency operation with low switching losses. The scheme structure is shown in Figure 2c.
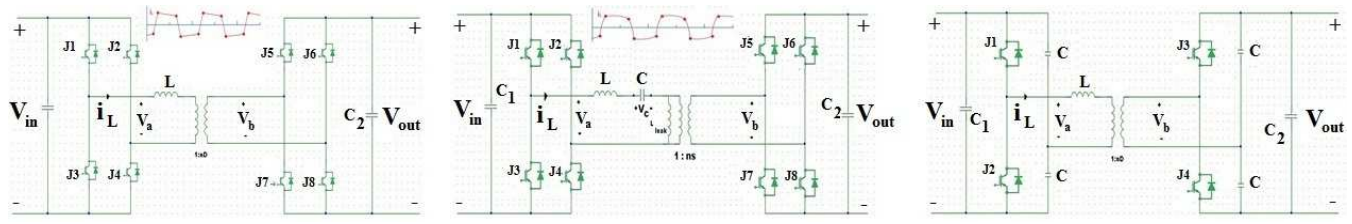

Figure 2: a) Structure of DAB. b) Structure of SRC. c) Structure of DHB. 
Table I: Advantages and drawbacks of each topology.

\begin{tabular}{c|c|c|c} 
& DAB & SRC & DHB \\
\hline Advantages & Electrical Insulation & Electrical Insulation & Electrical Insulation \\
& Soft switching in primary and secondary circuits & Soft switching in primary and secondary circuits & Soft switching both circuits \\
& Resonant inductance built into transformer & Sinusoidal currents & Uses less devices \\
& Less reactive power & Inductance leakage is not a problem & Transformer flux swing lower \\
\hline \multirow{2}{*}{ Drawbacks } & Soft switching may be lost at light load & Large resonant inductor (series inductance) & More reactive power \\
& Eight active power switches and control complex & High-voltage and high-current resonant capacitors & MOSFETs voltage limitation \\
& High ripple current in $C_{2}$ and large inductance leakage & Control complex & High current through the transformer \\
& Transformer saturation $\Rightarrow$ small capacitor in series & Complex control algorithm
\end{tabular}

\subsection{Multilevel converter}

One of the most important parts of power electronics converters are the semiconductor, because they must withstand high currents when they are "on" and large voltages when they are "off". If a large input/output voltage ratio is required, a single converter would not be enough, and an attractive solution is the interconnection of several converters modules or cells in series and parallel. This arrangement is commonly known as a multilevel converter. The multilevel solution adopted in this paper is illustrated in Figure 3. These types of converters are connected in multilevel structure for high power application, where each module is a bidirectional DC-DC converter constituting a bidirectional multilevel structure. This Multilevel structure consists of multiple low-voltage modules connected in series in one side and parallel in the other side.

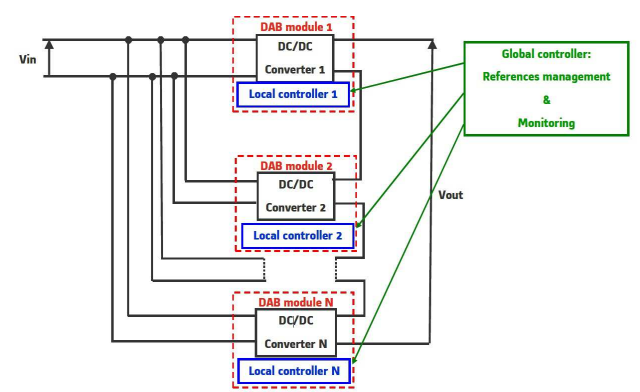

Figure 3: Building block multilevel DC transformer structure.

\subsection{Discussions and topology selection}

Three main topologies have been reviewed in this paper which all of them have advantages and drawbacks (see Table I). We will choose just one topology in order to perform DC/DC conversion and to test our future algorithms on DC grid. All of these topologies have been extensively tested and their performances experienced, so these topologies could be good candidates.

Since it uses fewer semiconductors, the DHB topology seems to be the most cost effective compared to DAB and SRC. However, the DHB topology have two main drawbacks: it is limited in power and high currents appear though the transformer and semiconductors in comparison with others. Moreover, the full-bridge topologies (DAB and SRC) allow applying zero volts to the transformer, so they have more degrees of freedom than the DHB topology. In this way, the voltage sources would provide less reactive power in the case of full bridge than the case of DHB topology. The main difference between the DAB topology and the SRC is that, in the SRC there is a capacitor in series with the inductance which does not exist in DAB configuration. The role of this capacitor is to resonate with the series inductance, reaching a sinusoidal current. Variable frequency is required in the SRC to obtain optimum performance. Consequently and thanks to its simplicity and cost efficiency compared to SRC, the DAB converter has been chosen to perform DC-DC conversion as single transformer module and in multilevel configuration for DC grid applications. 


\section{Dual Active Bridge (DAB). System Modeling}

As shown in Figure 2a, the DAB system which is based on power electronics is inherently discontinuous and consequently not adapted for control algorithm synthesis. In this work a continuous model is proposed. In order to simplify our circuit modeling, we will assume that the conduction losses are taken into account via small resistance $R_{\text {total }}$. In the modeling process, we will assume that the capacitors $C_{1}$ and $C_{2}$ are always precharged at the voltage levels $V_{\text {in }}$ and $V_{\text {out }}$. Therefore, these capacitors can be considered as ideal voltage sources with internal resistances (including in $R_{\text {total }}$ ). If all the parameters are seen from the primary side, we obtain the following simplified scheme as shown in Figure 4.

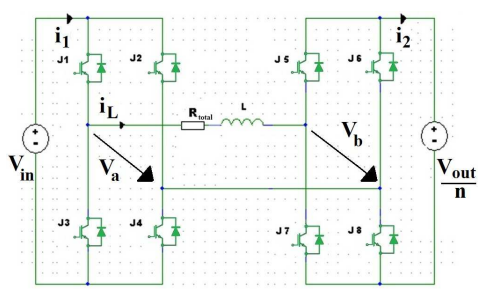

Figure 4: DAB module Simplified circuit.

To avoid short circuits and possible saturation of the transformer and the inductance, a zero average value of voltage is applied in each cycle. Consequently, a zero current average value is obtained in steady state. To achieve this claim, there exist several possibilities in the sequence of switching the power electronic components. Two interesting strategies have been shown in [6,14], where the authors have shown the advantages and drawbacks of each technique. In this proposed work, it is chosen to work with the method proposed in [14] (rectangular modulation), which ultimately is to play with the variable $d$ representing the phase shift ratio between the voltages applied to the inductance $V_{a}$ and $V_{b}$. In order to have a bidirectional converter, the phase shift ratio $d$ is defined in the interval $0<d<0.5$ for power flowing from left side to right side, and in the interval $-0.5<d<0$ for power flowing in the opposite direction.

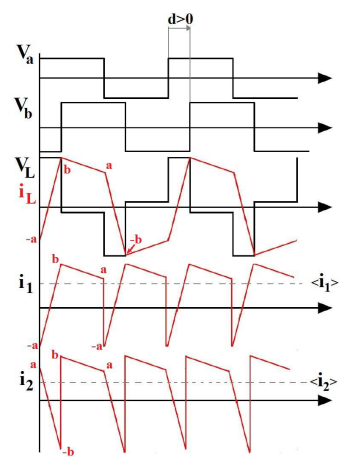

Figure 5: Voltages and currents in the DAB circuit $(d>0)$.

The applied voltages $V_{a}$ and $V_{b}$, the current $i_{L}$, the voltage $V_{L}$ in the inductance and currents $i_{1}$ and $i_{2}$ with their average values $\left\langle i_{1}\right\rangle$ and $\left\langle i_{2}\right\rangle$ (discontinuous lines) are obtained for a few cycles in (steady state) in Figure 5. It shows two interesting things. At first, and for the chosen phase shift ratio $(0<d<0.5)$, the average values of currents $\left(<i_{1}>\right.$ and $\left.\left.<i_{2}\right\rangle\right)$ are positive. This means that the power is flowing from the source $V_{\text {in }}$ to $V_{\text {out }}$. Secondly, we can remark that the average values of voltage and current applied to the inductance are zero. For all cases study, the following equations hold:

$$
\left\{\begin{array} { c } 
{ a = \frac { T } { 4 L } [ V _ { \text { in } } + ( 4 | d | - 1 ) \frac { V _ { \text { out } } } { n } ] } \\
{ b = \frac { T } { 4 L } [ ( 4 | d | - 1 ) \cdot V _ { \text { in } } + \frac { V _ { \text { out } } } { n } ] }
\end{array} \Rightarrow \left\{\begin{array}{c}
<i_{1}>=\frac{T}{L} \cdot d \cdot(1-2|d|) \frac{V_{\text {out }}}{n} \\
<i_{2}>=\frac{T}{n \cdot L} \cdot d \cdot(1-2|d|) V_{\text {in }}
\end{array}\right.\right.
$$




\subsection{Dynamics of the real system}

In order to design the nonlinear control based in Lyapunov theory a differential equation that adjusts to the real system is required. The control is performed on the mean value of current $i_{1}$, so all calculations based on this will be indicated with respect to the current $i_{1}$. The differential equation which governs the system is:

$$
\frac{d}{d t}<i_{1}>(t)=-\frac{R_{\text {total }}}{L} \cdot<i_{1}>(t)+\frac{T \cdot R_{\text {total }}}{L^{2}} \cdot d \cdot(1-2|d|) \frac{V_{\text {out }}}{n}
$$

Now, we are going to proof this equation 2 in the case of $0<d<0.5$. The case of negative $d$ is analogous. We have assumed that $V_{\text {in }}<\frac{V_{\text {out }}}{n}$, but if we chose the opposite, we can reach the same result. In $[0, d \cdot T]$ we have $\left(U=V_{\text {in }}+\frac{V_{\text {out }}}{n}\right)$ :

$$
\frac{d}{d t} i_{L}(t)=-\frac{R}{L} \cdot i_{L}(t)+\frac{U}{L} \Rightarrow b_{0}=i_{L}(d \cdot t)=a_{0} \cdot e^{-\frac{R}{L} d \cdot t}+\left(1-e^{-\frac{R}{L} d \cdot t}\right) \frac{V_{\text {in }}+\frac{V_{\text {out }}}{n}}{R}
$$

Now for $[d \cdot T, T / 2]$ we proceed the same way as above, but now $U=V_{\text {in }}-\frac{V_{\text {out }}}{n}$. So:

$$
a_{1}=i_{L}\left(\frac{T}{2}\right)=b_{0} \cdot e^{-\frac{R}{L}\left(\frac{1}{2}-d\right) T}+\left(1-e^{-\frac{R}{L}\left(\frac{1}{2}-d\right) T}\right) \frac{V_{\text {in }}-\frac{V_{\text {out }}}{n}}{R}
$$

Now is possible to put $a_{1}$ in terms of $a_{0}$, because $b_{0}$ depends on $a_{0}$, the expression is:

$$
a_{1}=a_{0} \cdot e^{-\frac{R T}{2 L}}+\left(1-e^{-\frac{R T}{2 L}}\right) \frac{V_{\text {in }}}{R}-\left(1-2 e^{-\frac{R}{L}\left(\frac{1}{2}-d\right) T+e^{-\frac{R T}{2 L}}}\right) \frac{V_{\text {out }}}{n \cdot R}
$$

As in the second part of the period the differential equations that govern the system are equal but with opposite voltages applied, proceeding in a similar way we obtain $a_{2}$ in terms of $a_{1}$ :

$$
a_{2}=a_{1} \cdot e^{-\frac{R T}{2 L}}-\left(1-e^{-\frac{R T}{2 L}}\right) \frac{V_{\text {in }}}{R}+\left(1-2 e^{-\frac{R}{L}\left(\frac{1}{2}-d\right) T+e^{-\frac{R T}{2 L}}}\right) \frac{V_{\text {out }}}{n \cdot R}
$$

Replacing equation 5 into 6, and put it in general terms we have:

$$
a_{n+2}+\left(1-e^{-\frac{R T}{2 L}}\right) a_{n+1}-\left(e^{-\frac{R T}{2 L}}\right) a_{n}=0
$$

That is a homogeneous linear recurrent succession with constant coefficients whose characteristic polynomial is:

$$
a_{n}=C t e_{1} \cdot(-1)^{n}+C t e_{2} \cdot e^{-n \frac{R T}{2 L}} \Rightarrow\left\{\begin{array}{c}
C t e_{1}=\frac{a_{0} \cdot e^{-\frac{R T}{2 L}}-a_{1}}{1+e^{-\frac{R T}{2 L}}} \\
\text { Cte } e_{2}=\frac{a_{0}+a_{1}}{1+e^{\frac{R T}{2 L}}}
\end{array}\right.
$$

The limit of the sub-succession of even terms and odd are shown in equations 9 and 10 respectively:

$$
\begin{aligned}
& \lim _{n \rightarrow+\infty} a_{2 n}=C t e_{1}=\frac{-\left[1-e^{-\frac{R T}{2 L}}\right] \frac{V_{\text {in }}}{R}+\left[1-2 e^{-\frac{R}{L}\left(\frac{1}{2}-d\right) T}+e^{-\frac{R T}{2 L}}\right] \frac{V_{\text {out }}}{n \cdot R}}{1+e^{-\frac{R T}{2 L}}} \\
& \lim _{n \rightarrow+\infty} a_{2 n+1}=-C t e_{1}=\frac{\left[1-e^{-\frac{R T}{2 L}}\right] \frac{V_{\text {in }}}{R}-\left[1-2 e^{-\frac{R}{L}\left(\frac{1}{2}-d\right) T}+e^{-\frac{R T}{2 L}}\right] \frac{V_{\text {out }}}{n \cdot R}}{1+e^{-\frac{R T}{2 L}}}
\end{aligned}
$$

It should be pointed out that the two expressions given above are independent of the initial values. Note that in steady $a_{2 n}$ and $a_{2 n+2}$ terms have the same value, and $a_{2 n+1}$ has the same value as $a_{2 n}$ and $a_{2 n+2}$ but with opposite sign. The mean value of the current $i_{1}$ in the interval $[n \cdot T,(n+1) \cdot T]$ is:

$$
<i_{1}>=c \cdot a_{2 n}+k(d) \Rightarrow\left\{\begin{array}{c}
c=\frac{L}{R T} \cdot\left(1-e^{-\frac{R T}{2 L}}\right) \\
k(d)=\left[1-\frac{3 L}{R T}\left(1-e^{-\frac{R T}{2 L}}\right)\right] \cdot \frac{v_{i n}}{R}+\left[4 d-1+\frac{3 L}{R T}\left(1-2 \cdot e^{-\frac{R}{L} \cdot\left(\frac{1}{2}-d\right) T}\right)\right] \cdot \frac{V_{o u t}}{n \cdot R}
\end{array}\right.
$$


If the periodic stepwise input signal does not change (voltage of sources), the dynamic of the succession of the values of $\left\langle i_{1}\right\rangle$ in each period is the same as the succession of their initial values $\left(a_{2 n}\right)$, so both have the same time constant $\tau$. For a first order system, it is by definition the time that the increase of the input signal reaches the value $\left(1-\frac{1}{e}\right)$ times the final increase value, when the input is constant. So:

$$
\left(\text { final value }-a_{0}\right)\left(1-\frac{1}{e}\right)=a_{2 n}-a_{0}
$$

The final value is obtained by the following expression:

$$
\lim _{n \rightarrow+\infty} a_{2 n}=\frac{a_{0} \cdot e^{-\frac{R T}{2 L}}-a_{1}}{1+e^{-\frac{R T}{2 L}}}
$$

We can obtain that $n \cdot T=\frac{L}{R}$, where $n$ is the number of periods for which the time constant reaches $\tau=n \cdot T$. Once we know the time constant, both for the succession of initial values and for the succession of average current values, is $\tau=\frac{L}{R}$, as the system for the current is a linear differential system of first order whose general expression is:

$$
\frac{d}{d t}<i_{1}>(t)=-\frac{<i_{1}>(t)}{\tau}+C t e
$$

Cte is a constant must be calculated. In equation 1, the average current in the steady state was calculated. Applying this result to equation 14 in steady state, one has:

$$
C t e=\frac{T}{\tau \cdot L} \cdot d \cdot(1-2|d|) \frac{V_{\text {out }}}{n}=\frac{T R}{L^{2}} \cdot d \cdot(1-2|d|) \frac{V_{\text {out }}}{n}
$$

So, we have demostrated that equation 14 has the same behaviour that equation 2 as we want to demonstrated $\square$.

\subsection{Verification of differential equation}

To verify that the differential equation have a properly behavior, we have created a file in SIMULINK to simulate the DAB bidirectional converter. The values of the simulation are shown in Table II

Table II: Verification of differential equation. Simulation values.

\begin{tabular}{|c|c|c|}
\hline $\mathrm{d}$ & Phase shift ratio & 0.15 \\
\hline $\mathrm{n}$ & Transformer ratio & $100 / 11$ \\
\hline$R_{\text {total }}$ & Total resistance & $0.01 \Omega$ \\
\hline$V_{\text {in }}$ & Input voltage & $1000 \mathrm{~V}$ \\
\hline$V_{\text {on }}$ & Output voltage & $10000 \mathrm{~V}$ \\
\hline $\mathrm{L}$ & Inductance & $6.875 \cdot 10^{-5} \mathrm{H}$ \\
\hline $\mathrm{T}$ & Period & $1 / 20000 \mathrm{~s}$ \\
\hline
\end{tabular}

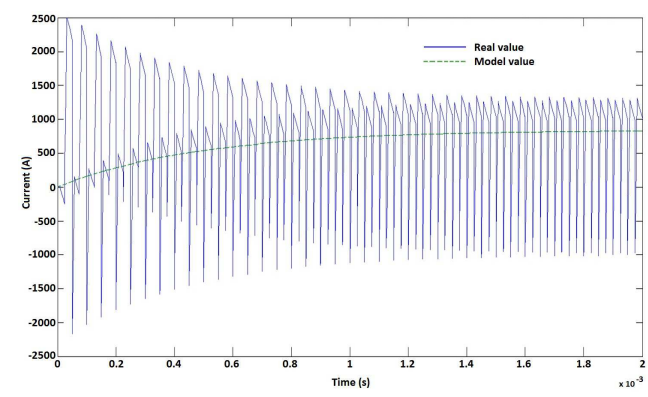

Figure 6: Comparison between real DAB (mean value $i_{1}$ ) and the dynamic average model for $\mathrm{d}=0.15$. 
In Figure 6 the solid plot corresponds to the real value of $i_{1}$ and the dashed plot is the output of our model corresponding to the differential equation 2. Figure 6 shows that the dynamic of the peaks of solid plot is the same as the dynamic of the dashed plot, so the transient behavior of the real current $i_{1}$ is the same that in the differential equation. In steady state the value of the average current $i_{1}$ is given by equation 1 which is the same as the steady state value in the differential equation 2 , so that it can be concluded that our model of equation differential is correctly modeled.

\subsection{Nonlinear control design for DAB system}

The proposed control law will be based on Lyapunov theory, see $[15,16]$. Consider the dynamic model represented in equation 2 Our aim is to track the reference of the average current $\left\langle i_{1}\right\rangle_{\text {ref }}$. Consider the following Lyapunov energy representing the needed energy necessary for the current to converge to its reference.

$$
Q_{<i_{1}>}=\frac{1}{2}\left(<i_{1}>-<i_{1}>_{r e f}\right)^{2}
$$

Its derivate time is:

$$
\frac{d}{d t} Q_{<i_{1}>}=E_{<i 1>} \cdot\left(-\frac{R}{L}<i_{1}>(t)+\frac{T R}{L^{2}} \cdot d \cdot(1-2|d|) \frac{V_{\text {out }}}{n}-\frac{d}{d t}<i_{1}>_{r e f}\right)
$$

where the $E_{<i 1>}=\left(<i_{1}>-<i_{1}>_{\text {ref }}\right)$ is the tracking error. In order to achieve to desired tracking as well as control stability, it is sufficient to force negative the derivative time of the Lyapunov function. In order to achieve negative derivate of the Lyapunov function:

$$
-\frac{R}{L}<i_{1}>(t)+\frac{T R}{L^{2}} \cdot d \cdot(1-2|d|) \frac{V_{\text {out }}}{n}-\frac{d}{d t}<i_{1}>_{r e f}=-\alpha_{<i 1>} \cdot E_{<i 1>}-\beta_{<i 1>} \cdot \operatorname{sign}\left(E_{<i 1>}\right)
$$

with the positive tuning gain parameters $\alpha_{<i 1>}>0, \beta_{<i 1>}>0$. As it is well known in the sliding mode control theory, the sign function is added to improve the robustness properties of the proposed control law. Thereafter, the time derivative of the Lyapunov function becomes:

$$
\frac{d}{d t} Q_{<i_{1}>}=-\alpha_{<i 1>} \cdot E_{<i 1>}^{2}-\beta_{<i 1>} \cdot \operatorname{sign}\left(E_{<i 1>}\right) \cdot E_{<i 1>} \leq 0
$$

Equation 19 is satisfying the stability condition. So, the following relation holds from equation 18 :

$$
d(1-2|d|)=\frac{n \cdot L^{2}}{R \cdot T \cdot V_{\text {out }}}\left[-\alpha_{<i 1>} E_{<i 1>}-\beta_{<i 1>} \operatorname{sign}\left(E_{<i 1>}\right)+\frac{d}{d t}<i_{1}>_{r e f}+\frac{R}{L}<i_{1}>\right]
$$

Let consider the right hand side of equation 20 as:

$$
K=\frac{n \cdot L^{2}}{R T V_{\text {out }}}\left[-\alpha_{<i 1>} E_{<i 1>}-\beta_{<i 1>} \operatorname{sign}\left(E_{<i 1>}\right)+\frac{d}{d t}<i_{1}>_{r e f}+\frac{R}{L}<i_{1}>\right]
$$

Developing equation 20, we obtain the general solution for the two cases leads to the control law which able to track the reference and to ensure the system stability:

$$
d(1-2|d|)=\left\{\begin{array}{l}
d-2 d^{2}, \text { for } d \geq 0 \\
d+2 d^{2}, \text { for } d \leq 0
\end{array} \Rightarrow d=\frac{1-\sqrt{1-8 K}}{4} \cdot S_{1}+\frac{-1+\sqrt{1+8 K}}{4} \cdot S_{2}\right.
$$

where $S_{1}$ has value 1 if the condition $K>0$ is true and 0 otherwise. The same applies for $S_{2}$ with the condition $K<0$.

\subsection{Real situation}

The goal of the converter is to be bidirectional, that is, it must be capable of changing the direction of the power at every instant. To do this, it is enough to change the reference current. The control is verified simulating a real situation where the current reference varies in the time. It should be noted that depending on the reference current imposed the power transmitted will be higher or lower. Figure 
7a shows that the system behaves well with respect to the different changes in the reference signal and Figure 7b shows that $d$ behaves correctly.
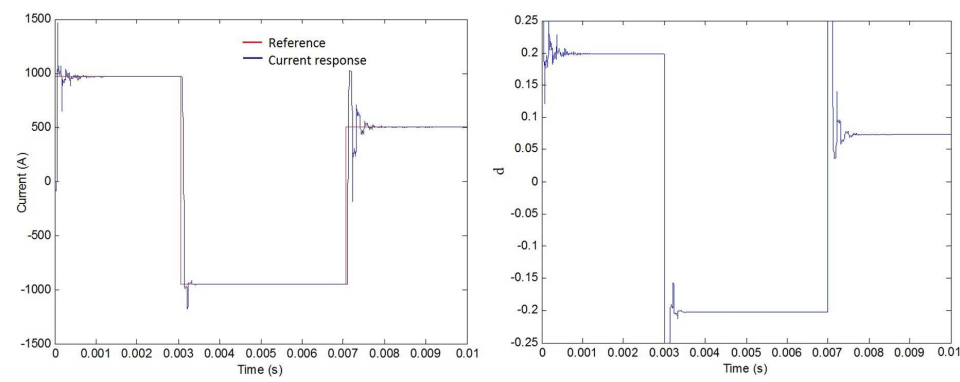

Figure 7: a) Current response b) $d$ response

\section{Multilevel Converter Control}

As shown in section 2.4, the proposed multilevel structure seems to be a good alternative for voltage elevation and multi-terminal DC grid interconnection. Once a robust control algorithm has been developed for DAB converter, our aim is to propose a multilevel "Plug \& Play" structure with DAB module association capable to raise the voltage output. It should be pointed out that the DAB module considered here is made of the DAB converter with its control system as shown in Figure 3.

The local controllers are designed as shown in section 3.3 and given by equation 22 . There is not need for additional control design and tuning parameters. The global controller (called also master controller) sends the requested references to each module in order to fulfill the global control requirements. If a failure occurs in a given module, the global controller will send an order to bypass the deteriorated module and keep the multilevel working in the voltage margin assigned by the operator. The deteriorated module can be replaced later with the global control acting in the same "Plug \& Play" philosophy. Once the regulation for a single module is obtained, the next step is to connect the modules as mentioned in section 2.4. This association of modules will help to obtain higher voltage ratios, without semiconductors suffering from it. Each module is controlled by the regulation obtained in the previous chapter. If $3 \mathrm{MW}$ want to be transmitted, emulating a wind turbine providing power to the network, with a ratio of $1 \mathrm{kV} / 150$ $\mathrm{kV}$, fifteen modules (with ratio $1 \mathrm{kV} / 10 \mathrm{kV}$ ) are connected, as it shows in Figure 8.

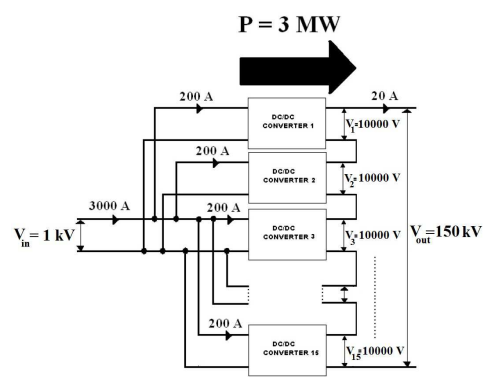

Figure 8: Multilevel converter with 3MW transmitted power

In this case the current reference in the primary of each module are $200 \mathrm{~A}$, so that the total current is $3000 \mathrm{~A}$, and with this current the desired power ( $3 \mathrm{MW}$ ) is obtained. The Figure 9a shows the current through the left side in each module. As shown in Figure 9a, in the primary of each module there are 200 $\mathrm{A}$, as expected. This means that our regulation is working properly. Thus we have that the total current through the low voltage side is $3000 \mathrm{~A}$, as seen in Figure $9 \mathrm{~b}$. But like this current is the current through the source $V_{i n}$, so total power supplied by this source is $3 \mathrm{MW}$, which is can be seen as a wind turbine. Figure $9 \mathrm{c}$ shows that current in the high voltage side is the current predicted. It is necessary also, to check the values of $d$ to check if it is correct for each module. As shown in Figure $9 \mathrm{~d}$ the value of $d$ is correct and it is stabilized at steady state. Now we check the value of high voltage side. 

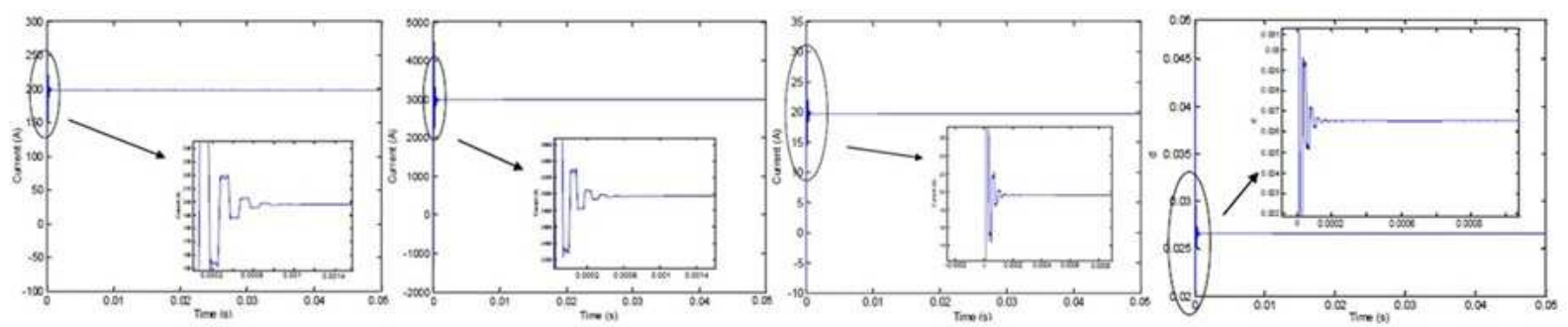

Figure 9: a) Current though the left side in the $n^{t} h$ cell b) Current in low voltage side c) Current in high voltage side d) $d$ response in $n^{t} h$ cell.

So we can conclude that our control for the multilevel converter $1 \mathrm{kV} / 150 \mathrm{kV}$ is a good solution to solve the problem. One of the main aspects of the proposed control is that the philosophy of control for a multilevel converter of $n$ modules is always the same, it consits in carry out an individual control of each module, with a current reference (could be different) for each module. The usual way of working is that all modules have the same reference, but there may be situations where different references are required; this is not a problem from the standpoint of control because, as said, control philosophy is individual for each module. A possible practical use is shown in Figure 10, which it shows an HVDC network composed of a solar installation, a wind farm and a storage. The HVDC network is connected to the global AC network. Individually control to each subsystem of HVDC network allows that the global system works satisfactory even if there is a failure in some subsystem. So the control is very robust, and it can be applied in many situations.

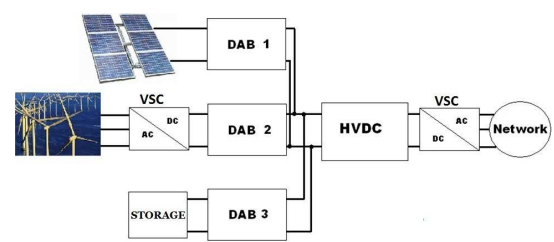

Figure 10: HVDC network

\section{Conclusions}

The main output of this paper is to provide DC/DC transformer solution renewable energy integration into multi-terminals DC grid. Two important requirements need to be satisfied: high voltage ratio between input and output DC transformer and bidirectional functionality of the converter. Three main topologies to perform DC/DC conversion have been reviewed in this work which all of them have advantages and drawbacks. Thanks to its simplicity and cost efficiency compared with others, the DAB converter has been chosen to perform DC-DC conversion as single transformer module and in multilevel configuration for DC grid applications. In this paper interesting multilevel approach has been proposed with multi-sources inputs where different technologies of power production and storage systems could be connected. This approach seems interesting in terms of coast and redundancy, since that each DC/DC transformer module is contributing to make the needed output voltage. Each module can contain the desired type of DC/DC bi-directional converter; however it is always advisable to have the same module in each converter, unless the structure has been developed in plug and play manner. All these advantages make the multilevel converter a good alternative in wind energy applications as it helps to fulfill the objectives of improved quality in the generated energy, and allows greater working powers to be reached whilst minimizing losses. Also, modelling and control of DAB converter have been proposed in this deliverable. Steady state average current analytic expression has given to help users in finding the current permanent values for all operations of the DAB converter. A piecewise averaging technique is adopted to model the DAB converter. This technique seems to be more suitable for our application. Based on this piecewise average dynamic model, nonlinear robust control law is derived. The obtained 
control algorithm has been tested in closed-loop, at first on the nonlinear average dynamic model, and then on the real electrical circuit representing the DAB converter. Simulation results have been presented to highlight the good performances of the proposed control algorithm. Once a robust control algorithm has been developed for DAB converter, our aim was to propose a multilevel "Plug \& Play" structure with DAB module association capable of elevating the voltage output. It should be pointed out that the DAB module considered here is made of the DAB converter with its control system. Simulation results in multilevel configurations have been proposed to show the effectiveness and performances in terms of dynamic response and robustness of the proposed control algorithm. The control design has been derived under the so-called "Plug \& Play" philosophy.

\section{References}

[1] J. Arrillaga, Y. Liu, and N. Watson, Flexible Power Transmission. The HVDC Options. John Wiley \& Sons Ltd, 2007.

[2] G. Asplund, "Ultra high voltaje trasmission," ABB review, February 2007.

[3] J. Kreuse, "The future is now," ABB review, March 2008.

[4] D. Ravemark and B. Normark, "Light and invisible," ABB review, March 2005.

[5] S. Johansson, G. Asplund, E.Jansson, and R. Rudervall, "Power system stability. benefits with vschvdc transmission systems," CIGRE, 2007.

[6] G.Ortiz, J. Biela, D. Bortis, and J. Kolar, "1 mw, $20 \mathrm{khz}$, isolated, 1.2-12kv bidirectional dc-dc renawable converter for energy applications," International Power Electronics Conference, IEEE, 2010 .

[7] G.Ortiz, J. Biela, and J. Kolar, "Optimized design of medium frequency transformers with high isolation requirements," 36th Conference on IEEE Industrial Electronics Society, November 2010.

[8] D. Aggeler, J. Biela, and J. Kolar, "Solid-state transformer based on sic jfet for future energy distribution systems," ETH Zurich, Power Electronic Systems Laboratory., 2009.

[9] J. Biela, D. Aggeler, D. Bortis, and J. W. Kolar, " $5 \mathrm{kv} / 200 \mathrm{~ns}$ pulsed power switch based on a sic-jfet super cascode," IEEE Power Modulators and High Voltage Conference, pp. 358-361, May 2008.

[10] P. Friedrichs, H. Mitlehner, R. Schorner, K. Donhke, R. Elpelt, and D. Stephani, "Stacked high voltage switch based on sic vjfets," ISPSD Cambridge, April.

[11] R. Singhi, "Ultra high voltage sic bipolar devices for reduced power electronics complexity," GeneSiC Semiconductor Inc, February.

[12] J. Palmour, "High voltage silicon carbide power devices," ARPA-E Power Technologies Workshop, CREE Inc, February.

[13] H.Fan and H. Li, "High frequency high efficiency bidirectional dc-dc converter module design for 10 kva solid state transformer," Applied Power Electronics Conference and Exposition (APEC), 2010 Twenty-Fifth Annual IEEE, vol. 1, pp. 210-215, February 2010.

[14] J.Sebastián, A.Rodríguez, D. G. Lamar, M. M. Hernando, and A. Vázquez, "An overall study of a dual active bridge for bidirectional dc/dc conversion," Energy Conversion Congress and Exposition, pp. 1129-1135, 2010.

[15] A. Isidori, "Nonlinear control systems," Springer Verlag, 1995.

[16] H. K. Khalil, “Nonlinear systems,” Prentice Hall, 2002. 